\title{
Gamma-delta T cells in childhood acute lymphoblastic leukemia act as an early marker of favorable prognosis and correlate with serum HSP90.
}

\author{
Dorota Pawlik-Gwozdecka ${ }^{1}$, Maciej Zielinski ${ }^{1}$, Justyna Sakowska ${ }^{1}$, Magdalena \\ Górska-Ponikowska ${ }^{1}$, Elżbieta Adamkiewicz-Drożyńska ${ }^{1}$, Piotr Trzonkowski ${ }^{1}$, and Maciej \\ Niedźwiecki ${ }^{1}$ \\ ${ }^{1}$ Medical University of Gdansk
}

May 29, 2020

\begin{abstract}
Acute lymphoblastic leukemia (ALL) is the leading cause of cancer related death in children despite recent advances showing improved responses to chemotherapy treatment. Gamma-delta $(\gamma \delta) \mathrm{T}$ cells are a recent topic of growing interest in the field of adoptive immunotherapy. These cells have also been proven to be an optimal predictor of a favorable outcome in numerous malignancies. We evaluated subgroups of $\gamma \delta \mathrm{T}$ cells in the peripheral blood of 19 children with newly diagnosed ALL at the time of diagnosis and following chemotherapy induction. Due to the fact that serum HSP90 serves as a ligand for $\gamma \delta$ T cells, we also checked the correlation between HSP90 and $\gamma \delta$ T cells. As a result, we found, that the number of CD8+ $\gamma \delta$ T cells in peripheral blood at disease presentation was almost three times higher in the intermediate risk group compared to patients in high risk group. Furthermore, we observed higher percentages of the subset in younger patients at diagnosis and after induction, but not in healthy controls. We also noticed negative correlations between CD $8+\gamma \delta \mathrm{T}$ cells and minimal residual disease (MRD) before chemotherapy and after induction. We showed a positive association between activated CD3+ $\gamma \delta$ T cell and serum HSP90 at presentation. In conclusion, our data suggests that CD8+ $\gamma \delta \mathrm{T}$ cells may be an early marker of favorable prognosis in childhood ALL while serum HSP90 may act as an agent activating CD3+ ró T effector cells.
\end{abstract}

\section{Hosted file}

Gdtcells_Final_CEI.pdf available at https://authorea.com/users/327680/articles/455152gamma-delta-t-cells-in-childhood-acute-lymphoblastic-leukemia-act-as-an-early-marker-offavorable-prognosis-and-correlate-with-serum-hsp90 